\title{
FIRST LASING OF A HIGH-GAIN HARMONIC GENERATION FREE- ELECTRON LASER EXPERIMENT*
}

L.-H. Yuं, M. Babzien, I. Ben-Zvi, L. F. DiMauro, A. Doyuran, W. Graves, E. Johnson, S. Krinsky, R. Malone, I. Pogorelsky, J. Skaritka, G. Rakowsky, L. Solomon, X.J. Wang, M. Woodle, V. Yakimenko

Brookhaven National Laboratory, Upton, New York 11973

S.G. Biedron, J.N. Galayda, E. Gluskin, J. Jagger, V. Sajaev, I. Vasserman Advanced Photon Source, Argonne National Laboratory, Argonne, Illinois 60439

Abstract

We report on the first lasing of a high-gain harmonic generation (HGHG) free-electron laser (FEL). The experiment was conducted at the Accelerator Test Facility (ATF) at Brookhaven National Laboratory (BNL). This is a BNL experiment in collaboration with the Advanced Photon Source (APS) at Argonne National Laboratory. A preliminary measurement gives a high-gain harmonic generation (HGHG) pulse energy that is $2 \times 10^{7}$ times larger than the spontaneous radiation. In a purely self-amplified spontaneous emission (SASE) mode of operation, the signal was measured as 10 times larger than the spontaneous radiation in the same distance $(\sim 2 \mathrm{~m})$ through the same wiggler. This means the HGHG signal is $2 \times 10^{6}$ times larger than the SASE signal. To obtain the same saturated output power by the SASE process, the radiator would have to be 3 times longer $(6 \mathrm{~m})$.

* Work supported by U.S. Department of Energy, Office of Basic Energy Sciences, under Contracts Nos. DEAC02-98CH10886 and W-31-109-ENG-38 and by Office of Naval Research Grant no. N00014-97-1-0845. 


\section{DISCLAIMER}

This report was prepared as an account of work sponsored by an agency of the United States Government. Neither the United States Government nor any agency thereof, nor any of their employees, make any warranty, express or implied, or assumes any legal liability or responsibility for the accuracy, completeness, or usefulness of any information, apparatus, product, or process disclosed, or represents that its use would not infringe privately owned rights. Reference herein to any specific commercial product, process, or service by trade name, trademark, manufacturer, or otherwise does not necessarily constitute or imply its endorsement, recommendation, or favoring by the United States Government or any agency thereof. The views and opinions of authors expressed herein do not necessarily state or reflect those of the United States Government or any agency thereof. 


\section{DISCLAIMER}

Portions of this document may be illegible in electronic image products. Images are produced from the best available original document. 


\section{Introduction}

At the Accelerator Test Facility (ATF) at Brookhaven National Laboratory (BNL), a freeelectron laser (FEL) experiment based on the high-gain harmonic generation (HGHG) principle [1,2] has achieved first lasing at $5.3 \mu \mathrm{m}$. In HGHG, a coherent seed at a wavelength at a subharmonic of the desired output radiation interacts with the electron beam in an energy-modulating section. This energy modulation is then converted into spatial bunching while traversing a dispersive section (a three-dipole chicane). In the second undulator (the radiator), which is tuned to a higher harmonic of the seed radiation, the microbunched electron beam first emits coherent radiation and then amplifies it exponentially until saturation is achieved. Harmonic generation using a seed laser is well known and has been verified experimentally and analyzed [3]. However, HGHG, i.e., harmonic generation followed by an exponential growth to achieve saturation is realized for the first time in our new experiment. Here, a description of the HGHG experiment and the preliminary results will be discussed.

\section{The Experiment}

A schematic of the HGHG apparatus is illustrated in Figure 1. The existing ATF photocathode rf gun, linac, and coherent seed radiation source, a $\mathrm{CO}_{2}$ laser, define the electron and seed beam design parameters found in Figure 1. The value of the energy was specifically tailored to achieve the FEL resonance condition with an existing radiator section, provided by Cornell University and modified and measured by the Advanced Photon Source (APS). The modulator section and dispersive sections for HGHG operation were designed, manufactured, and measured at BNL. These magnetic 
component parameters are also found in Figure 1. Based on these design parameters, the output power as predicted by theory and simulation is $35 \mathrm{MW}$ in $2 \mathrm{~m}$ of the radiator (see Figure 2).

On July 15,1999, SASE was measured at $5.3 \mu \mathrm{m}$. In a one-week period, we carried out several sets of measurements at different currents and emittances. For example, the results of one experiment had a current of $120 \mathrm{~A}(0.8 \mathrm{nC}$ in $6 \mathrm{ps}$ FWHM) with an emittance of $5.5 \mathrm{~mm}$-mrad and a global energy spread of $\sim 0.6 \%$. The measured ratio of SASE to spontaneous radiation for this case was 13.6 and can be compared to the theoretical ratio of 13.2. All of these SASE measurements were taken with a bandpass filter at $5.3 \mu \mathrm{m}$ with $2 \%$ bandwidth and an InSb point detector. The set of measured SASE over spontaneous ratios are plotted in Figure 3, along with the design and compared with theory. The solid lines were calculated using an analytical formula [4]. The good agreement between the experimental data and theory gives us great confidence in the reliability of the current and emittance measurements. From these results we determined to operate our HGHG experiment around $120 \mathrm{~A}$ and $5.5 \mathrm{~mm}$-mrad.

Over the following two weeks, we turned on the dispersion section and closed the gap of the mini-undulator (the modulator) to be resonant at $10.6 \mu \mathrm{m}$, and we corrected the trajectory to compensate for these changes [5].

On August 7, 1999, we began seeding with the $\mathrm{CO}_{2}$ laser. Adjustment of the optical trombone length synchronizes the electron beam and the $\mathrm{CO}_{2}$ laser, thus creating an 
energy modulation. The modulated beam passing through a dipole (part of the spectrometer) generates at the end of the HGHG beamline an electron beam profile whose horizontal axis corresponds to the energy spread. In Figures $4 \mathrm{a}$ and $4 \mathrm{~b}$, the horizontal distribution corresponds to the energy distribution without and with energy modulation, respectively. By adjusting the optical trombone length, we can vary the $\mathrm{CO}_{2}$ arrival time relative to the electron beam. The square of the energy modulation is proportional to the $\mathrm{CO}_{2}$ power. When the energy modulation squared is plotted versus the delay time in the optical trombone, the $\mathrm{CO}_{2}$ pulse width was determined to be $\sim 300 \mathrm{ps}$ as seen in Figure 5. Combined with the $\mathrm{CO}_{2}$ pulse energy measurement, this yields a $\mathrm{CO}_{2}$ power of $500 \mathrm{MW}$. We attenuated the laser beam by a factor of three to protect the input window and obtained a large energy modulation as shown in Figure 4b.

From August $11-20,1999$, we had eight days to measure HGHG. Each day we routinely aligned the $\mathrm{CO}_{2}$ laser for energy modulation and carried out a SASE measurement by simply not triggering the $\mathrm{CO}_{2}$ laser. The SASE power at $5.3 \mu \mathrm{m}$ as a function of charge in one of the measurements is shown in Figure 6. For the maximum charge in this SASE measurement $(\sim 0.8 \mathrm{nC})$, the detector signal is shown in Figure 7 . When we attenuated the $\mathrm{CO}_{2}$ laser by a factor of 1000 (attenuated to a power of about $0.5 \mathrm{MW}$ ) and placed $10^{6}$ attenuation in front of the same InSb detector and then triggered the $\mathrm{CO}_{2}$ laser, the HGHG signal at this same charge was measured and is shown in Figure 8. Notice that the HGHG signal with attenuation is three times larger than the SASE signal without attenuation. Therefore, the HGHG signal is $3 \times 10^{6}$ larger than the SASE signal in the same length of undulator $(1.98 \mathrm{~m})$. The attenuation of $10^{6}$ was based on the specification of the manufacturer, hence we still need to verify this further by our own calibration. 
Based on these sets of measurements, we know that the pulses have energies on the order of tens of $\mu \mathrm{J}$. A Pyroviewer thermal imager was then used to measure the transverse profile of the output radiation as shown in Figure 9. The measurement was performed using two magnesium fluoride short-pass filters for blocking the $\mathrm{CO}_{2}$ laser and a $2 \%$ bandpass filter at $5.3 \mu \mathrm{m}$. Additional tests were provided by recording the image for each of the following conditions: 1) the electron beam off with the $\mathrm{CO}_{2}$ laser on, 2) the $\mathrm{CO}_{2}$ laser off and the electron beam on. Both cases produced no image except background. This confirms that the image depends on the presence of both the electron beam and $\mathrm{CO}_{2}$ laser. Finally, to further confirm the radiation is indeed the $5.3 \mu \mathrm{m}$ radiation, the abovedescribed bandpass filter was removed and the image barely changed. In addition, the uniformity of the transverse profile indicates excellent transverse coherence, although this must be verified in the future.

On August 17, we measured the HGHG pulse energy directly using a Joule meter. The maximum output of the Joule meter during this run was $65 \mu \mathrm{J}$, as shown in Figure 10. During this run, we also measured the electron beam pulse length to be $\sim 6$ ps FWHM. If we assume the radiation pulse is also 6 ps (actually, it is more likely to be shorter), then the output would be $11 \mathrm{MW}$. Since we know the spontaneous radiation power is $0.5 \mathrm{~W}$, as confirmed by measurement within the observation solid angle at the InSb detector and by theory, we again show the HGHG output is $2 \times 10^{7}$ times larger than the spontaneous power. 
On August 19, 1999, we measured the first HGHG spectrum. The result is shown in Figure 11. The HGHG output radiation is sent through a beam-splitter, one part is sent to a Joule meter and the other through the spectrometer. For each individual shot, the output of the spectrometer is divided by the output of the Joule meter, and this normalized power spectrum is plotted as a function of wavelength. This figure shows a bandwidth of $\sim 20$ $\mathrm{nm}$. The large fluctuation in the spectrum indicates that the spectrum changes from shot to shot, even though it remains in the bandwidth of $20 \mathrm{~nm}$. This fluctuation is not expected from HGHG theory and might be due to e-beam pulse shape change; it needs to be further studied. If this pulse is Fourier transform limited, this $20 \mathrm{~nm}$ bandwidth can be used to calculate the radiation pulse length, which is about $1.5 \mathrm{ps}$. We are presently preparing a pulse-length measurement, if we can confirm the $1.5 \mathrm{ps}$ duration, then the peak power would be $44 \mathrm{MW}$. This number would be closer to the theoretically predicted $35 \mathrm{MW}$. The SASE spectrum from the same radiator, multiplied by a factor of $10^{6}$, and the HGHG spectrum, are shown in Figure 11 for comparison. The SASE bandwidth is six times larger than the HGHG bandwidth.

With attenuation of 1000 times, the $\mathrm{CO}_{2}$ power is on the order of $0.5 \mathrm{MW}$. At this power, we found the HGHG signal was maximized. The energy-modulation diagram obtained using the electron energy spectrometer, as described above, is shown for this condition in Figure $4 \mathrm{c}$. This shows an energy modulation of nearly $1 \%$, which is equal to the Pierce parameter, which is $\sim 0.009$, indicating that the system was saturated. If we compare this value with the theoretical calculations of the phase-space distribution at the exit of the 
radiator shown in Figure 12, we can immediately recognize the double-band structure in Figure 4c. This corresponds to the particles trapped in the phase-space bucket.

On August 20,1999, we measured the HGHG output energy as a function of the $\mathrm{CO}_{2}$ power, as shown in Figure 13. When we compare this with the theoretical value of the output HGHG power versus $\mathrm{CO}_{2}$ power, we find a clear similarity. The quantitative comparison of these two curves is not warranted because the experimental conditions are not exactly the same as the design conditions.

\section{Conclusions}

The preliminary results of the first lasing of the HGHG experiment at the ATF have confirmed some of the theoretical predictions. The SASE results are in good agreement with theory, seeding with the $\mathrm{CO}_{2}$ laser produced the anticipated energy modulation, and HGHG was demonstrated by converting $10.6 \mu \mathrm{m}$ seed into high power $5.3 \mu \mathrm{m}$ radiation. This output was $2 \times 10^{7}$ larger than the spontaneous emission.

\section{References}

[1] I. Ben-Zvi, L.F.DiMauro, S. Krinsky, M.G. White, L.H. Yu, Nucl. Instr. Meth. A304, $151(1991)$.

[2] L. H. Yu, Phys. Rev A 44, 5178 (1991); I. Ben-Zvi et al., Nucl. Instrum. Meth. A318, 208 (1992).

[3] R. Prazeres, et al., Nucl. Instr. Meth. A304, 72 (1991); I. Boscolo and V. Stagno, Nucl. Instr. Meth. 188, 483 (1982); R. Bonifacio, L. de Salvo Souza, P. Pierini and E.T. Scharlemann, Nucl. Instr. Meth. A296, 787 (1990). 
[4] L.-H. Yu, Phy. Rev. E 58, 4991 (1998); L.H.Yu, Proceedings of FEL Conference 1998, Williamsburg.

[5] V. Sajaev, L.-H. Yu, A. Doyuran, R. Malone, X. Wang, V. Yakimenko, "Diagnostics and Correction of the Electron and Beam Trajectory in the Cornell Wiggler at the Accelerator Test Facility," Proceedings of the 1999 Particle Accelerator Conference, New York, NY, March 29-April 2, 1999 (to be published). 


\section{LIST OF FIGURES}

Figure 1: HGHG experiment schematic.

Figure 2: Simulation of HGHG output as a function of wiggler distance.

Figure 3: Ratio of SASE over spontaneous radiation power measured, compared with the analytic theory, and designed value.

Figure 4a: Energy spread without energy modulation; 4b energy modulation generated by $\mathrm{CO}_{2}$ power of about $150 \mathrm{MW}$; $4 \mathrm{c}$ : energy modulation with $\mathrm{CO}_{2}$ power of about $0.5 \mathrm{MW}$ and HGHG lasing to saturation.

Figure 5: $\mathrm{CO}_{2}$ power as a function of time, measured by optical trombone.

Figure 6: The SASE signal versus charge.

Figure 7: SASE signal from the InSb detector.

Figure 8: HGHG signal from the InSb detector with $10^{6}$ attenuation.

Figure 9: HGHG output radiation profile by a Pyroviewer thermal camera.

Figure 10: HGHG output signal from a Joule meter. 
Figure 11: HGHG power spectrum (resolution $5 \mathrm{~nm}$ ) plotted with SASE spectrum measured using the same detector but multiplied by a factor $10^{6}$.

Figure 12: Phase-space diagram showing large energy modulation at the exit of the radiator.

Figure 13: HGHG output energy vs. $\mathrm{CO}_{2}$ power, as compared with theory. 


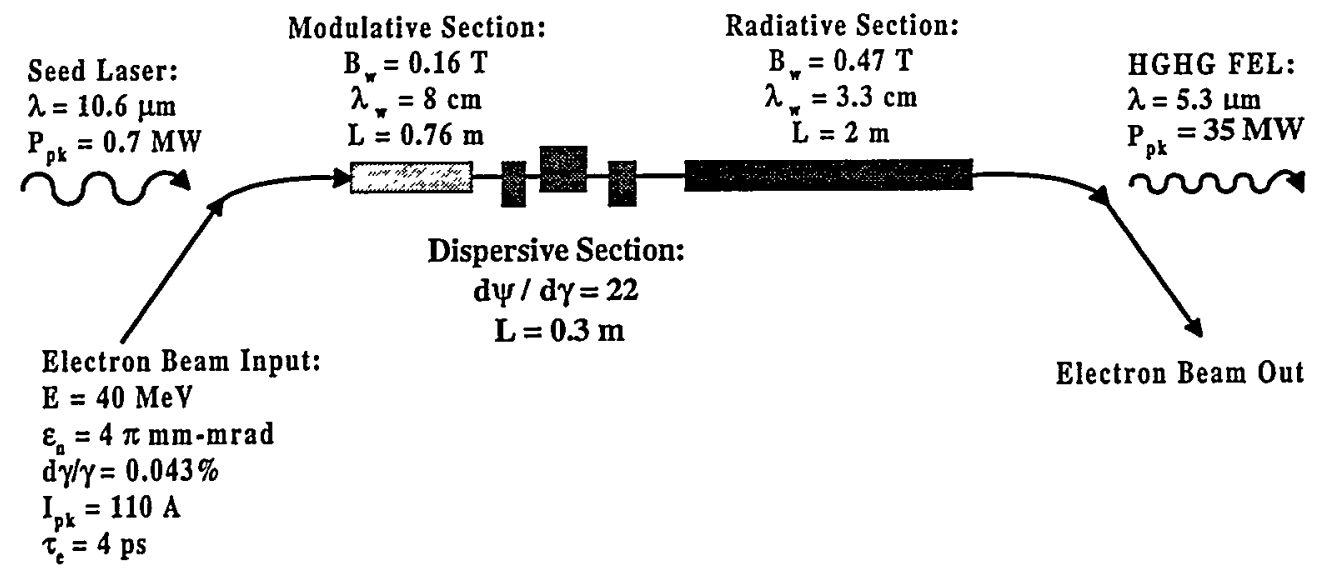

Figure 1 


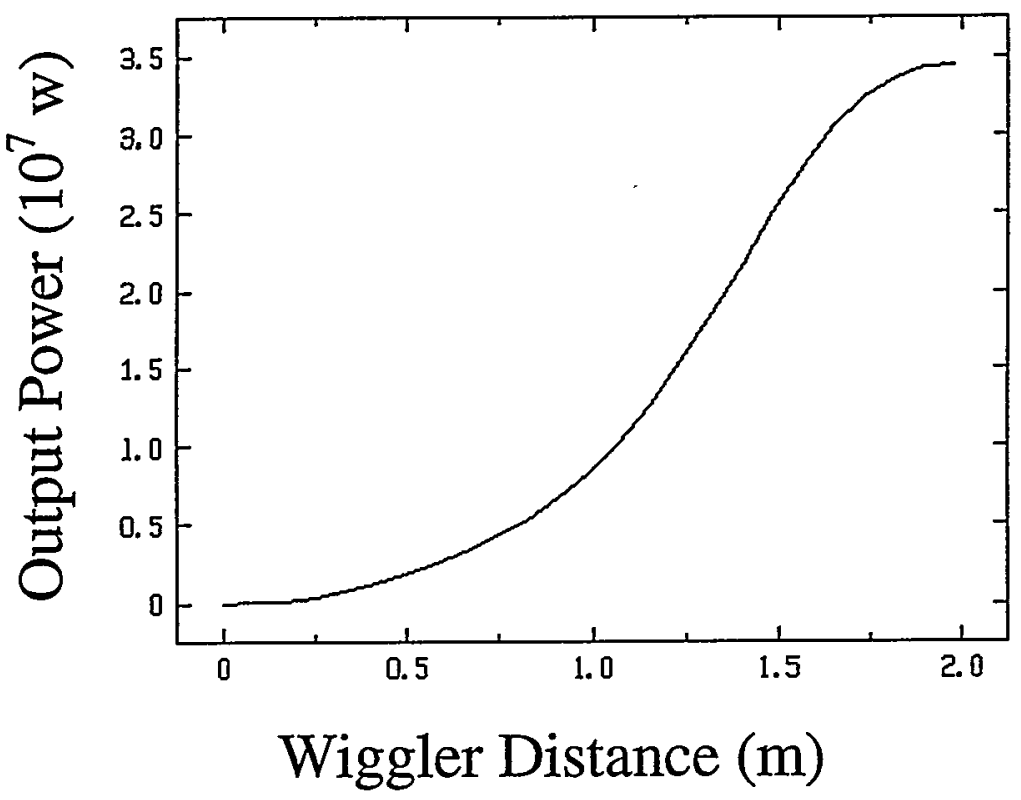

Figure 2 


\section{SASE/spontaneous power ratio}

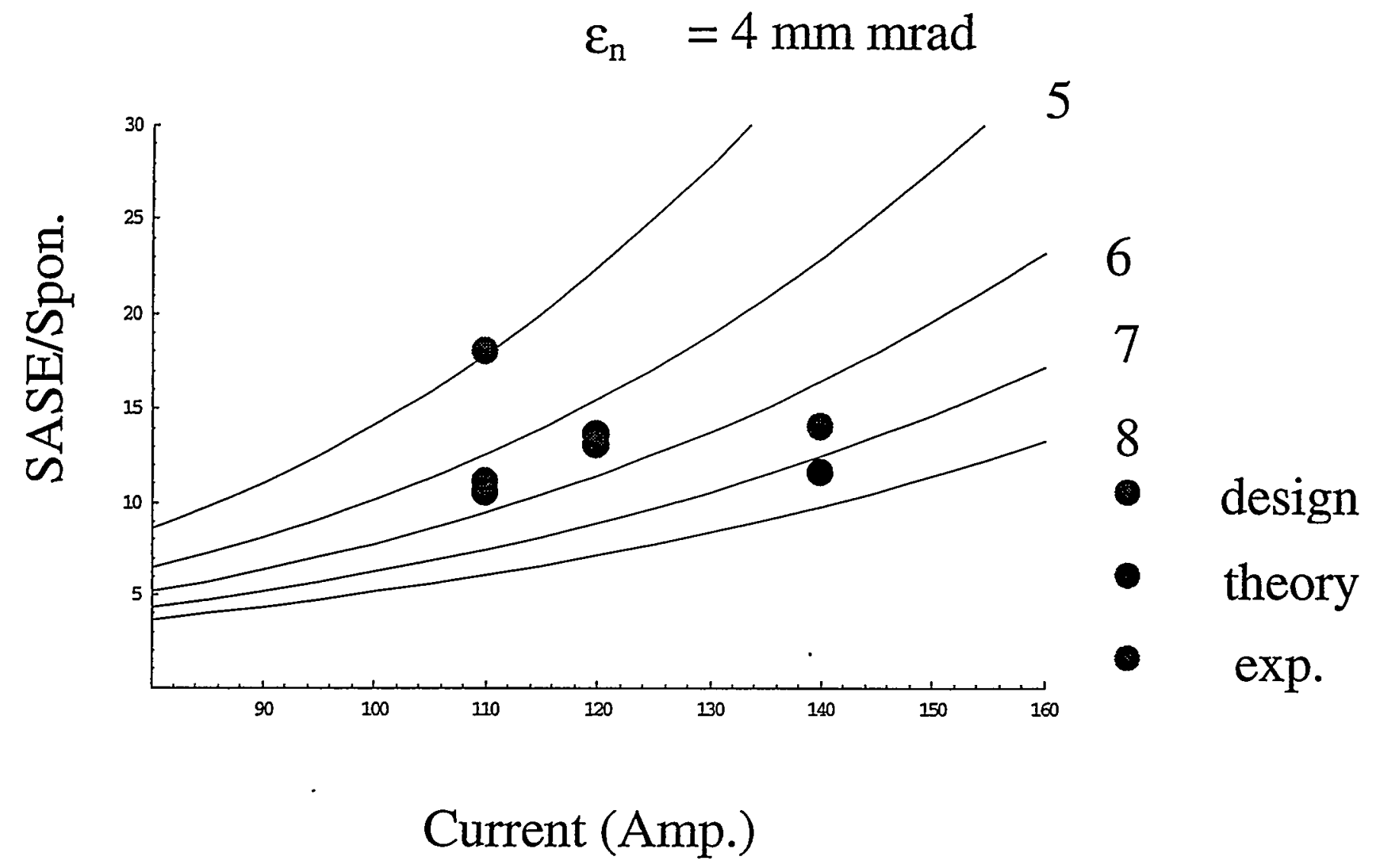

Figure 3 


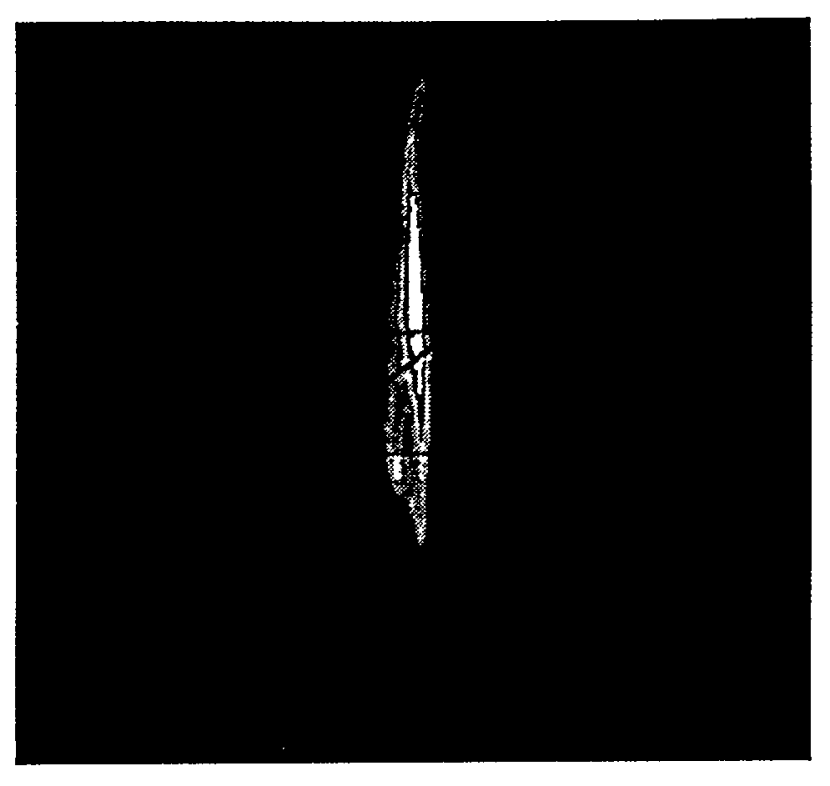

Figure 4a

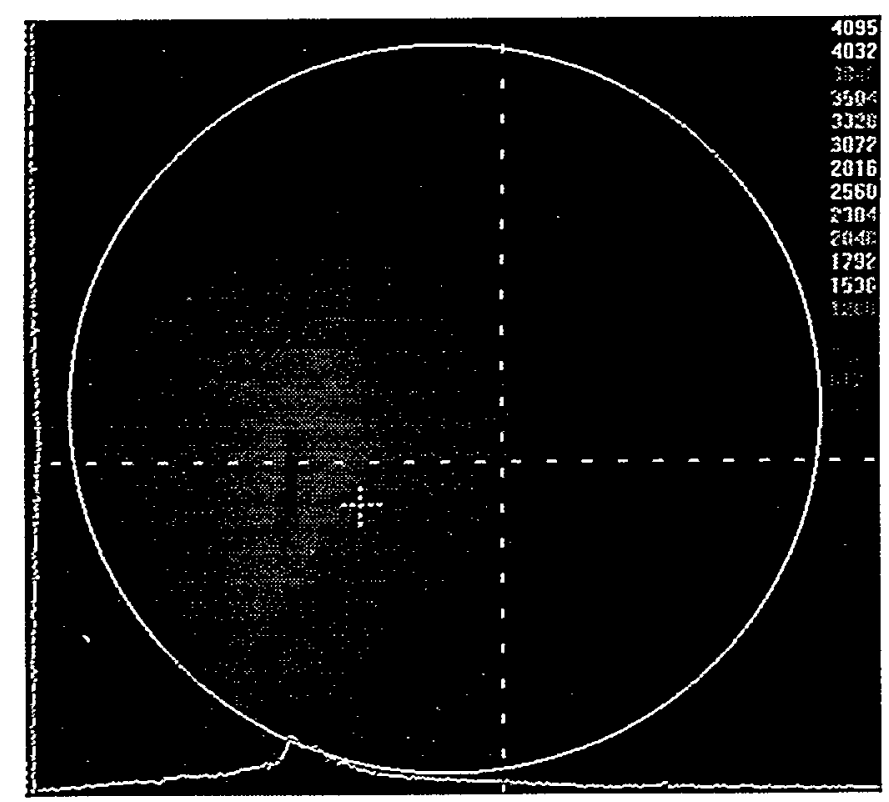

Figure 4b 


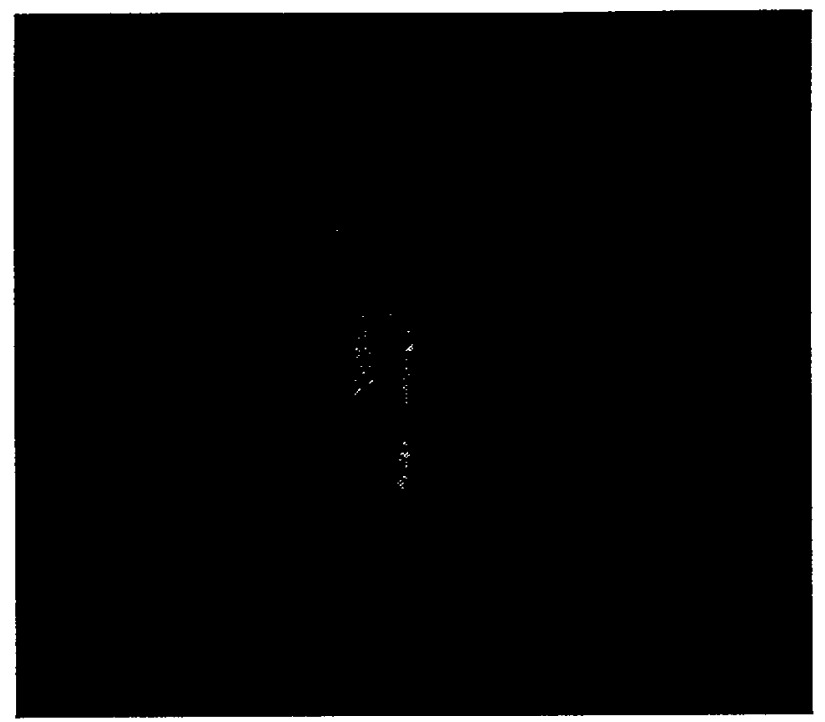

Figure 4c 


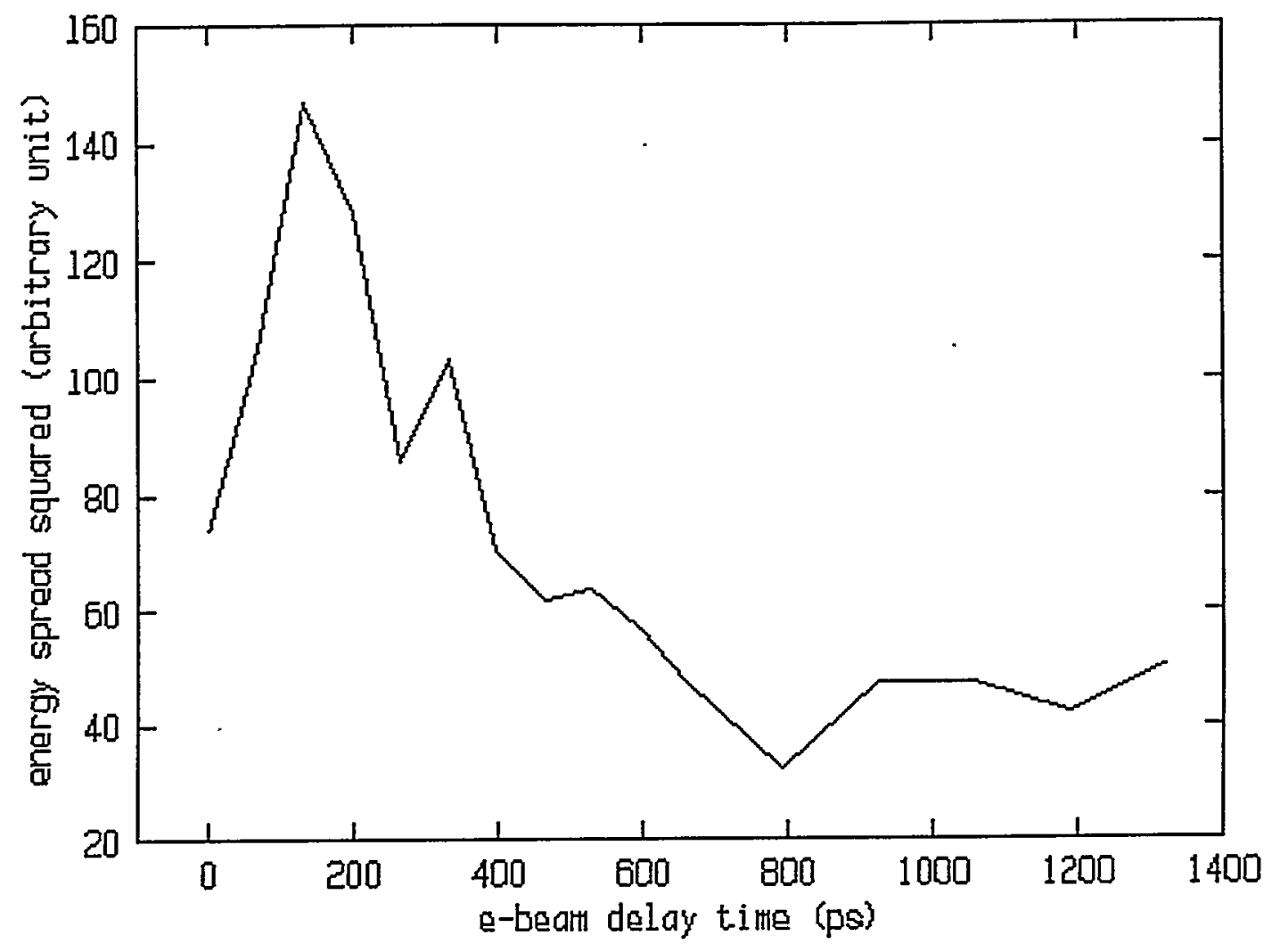

Figure 5. 


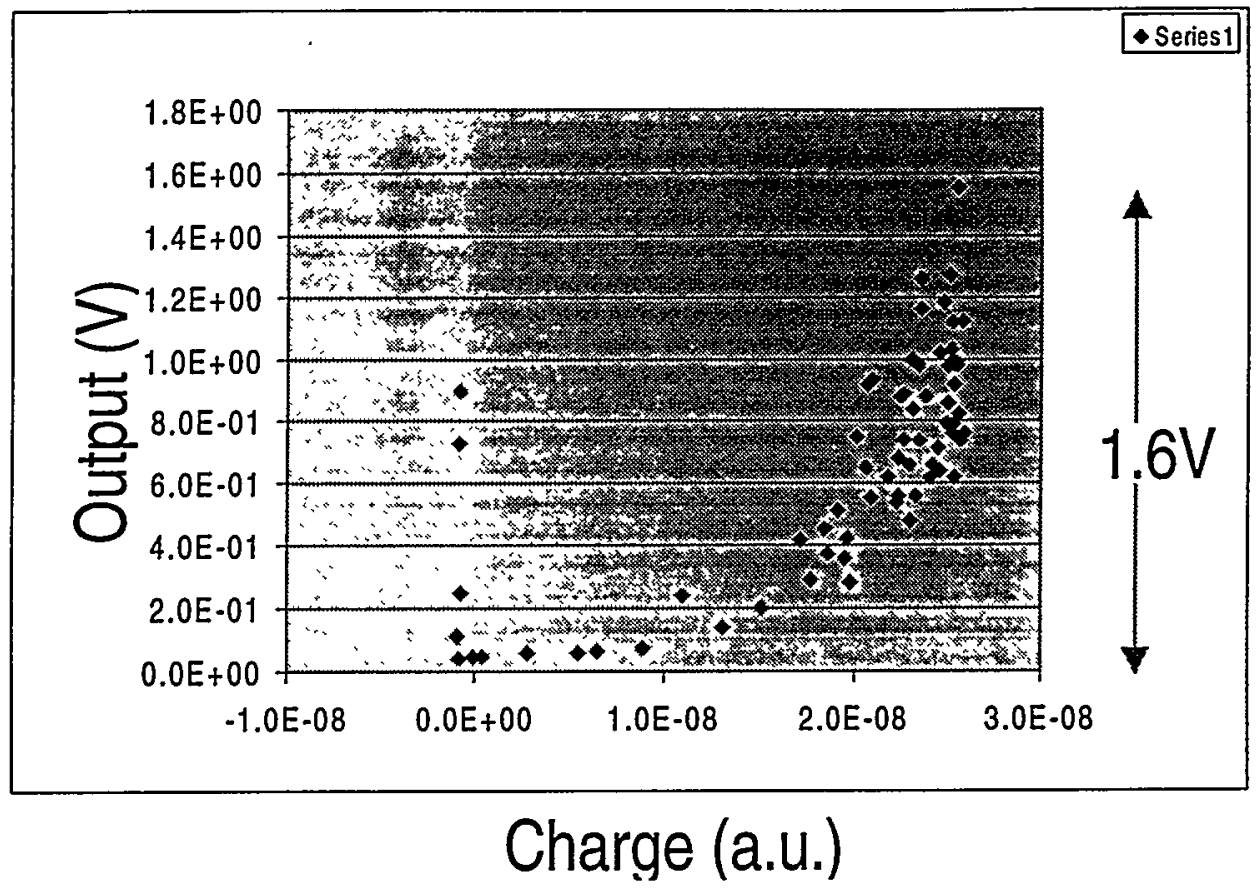

Figure 6 


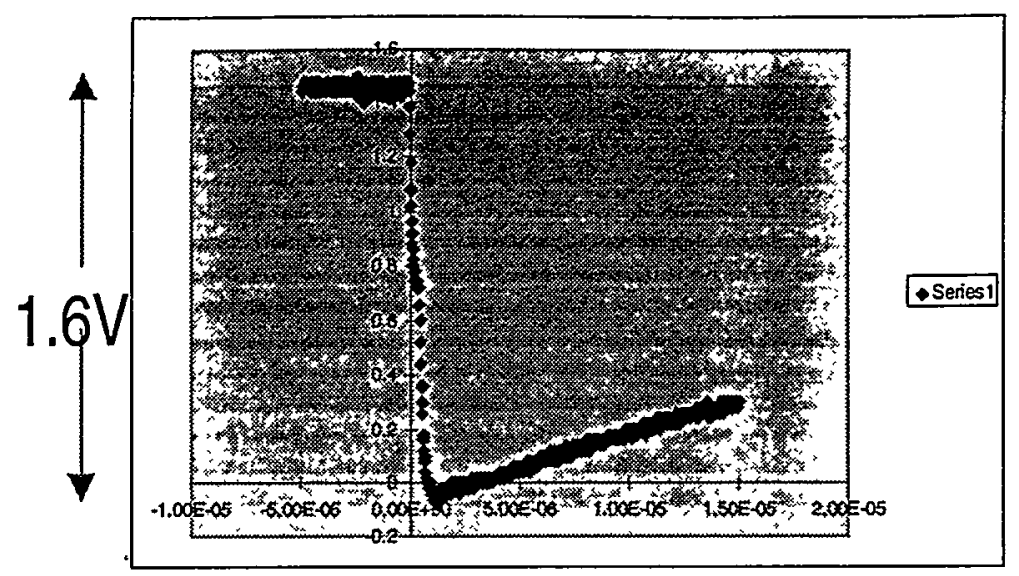

Figure 7

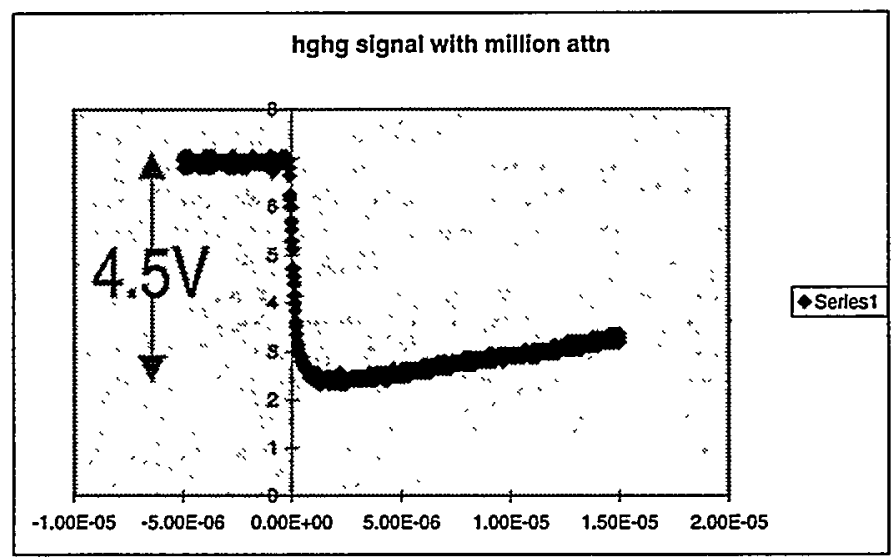

Figure 8 


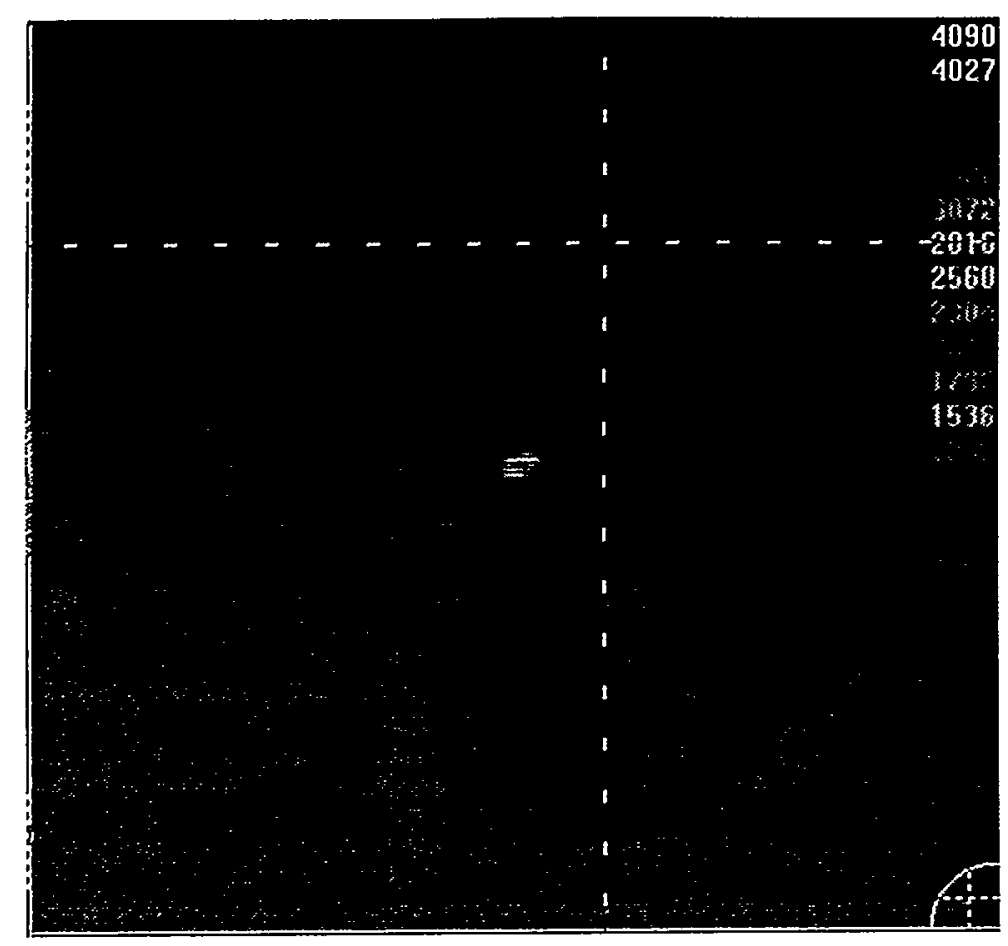

Figure 9. 


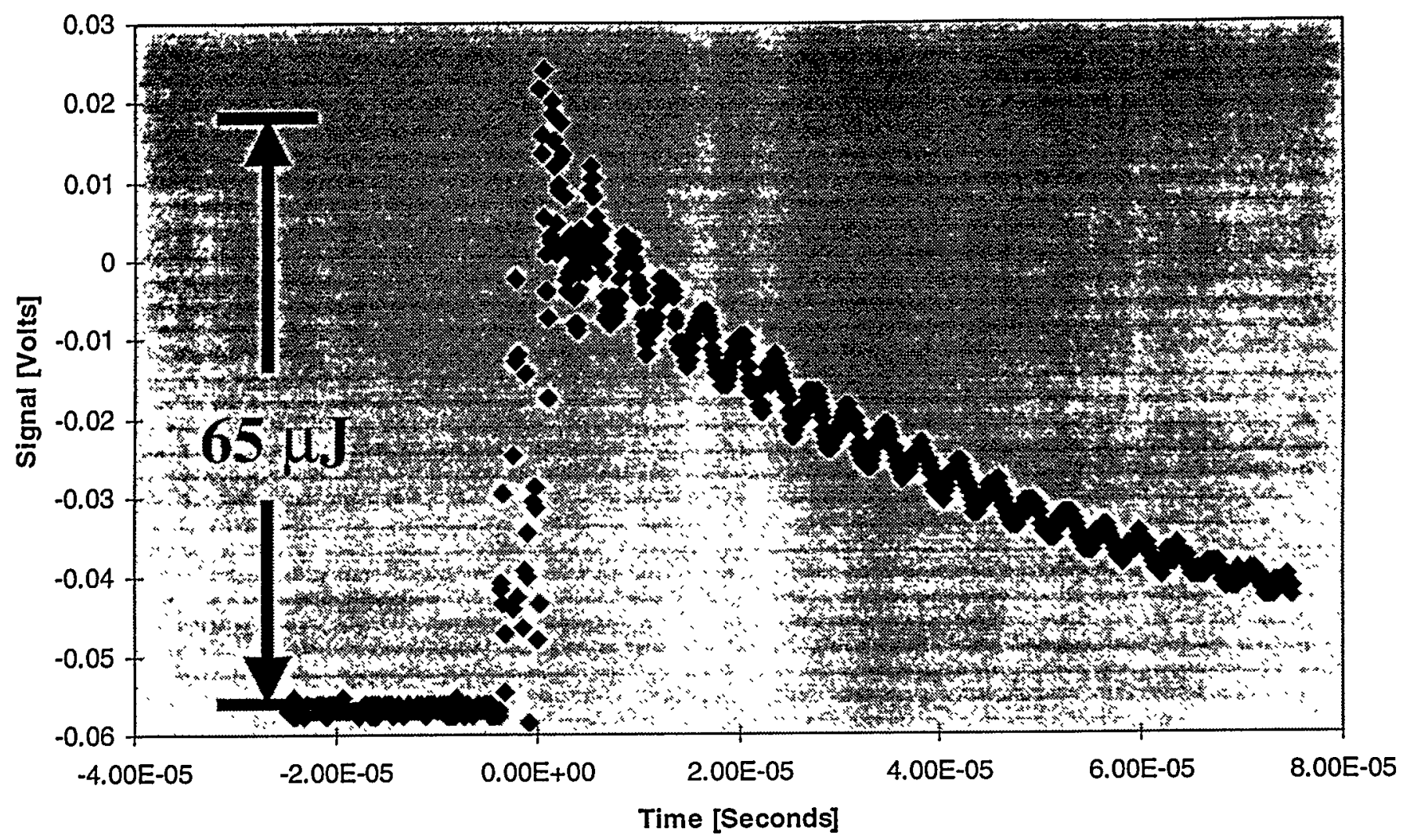

Figure 10 


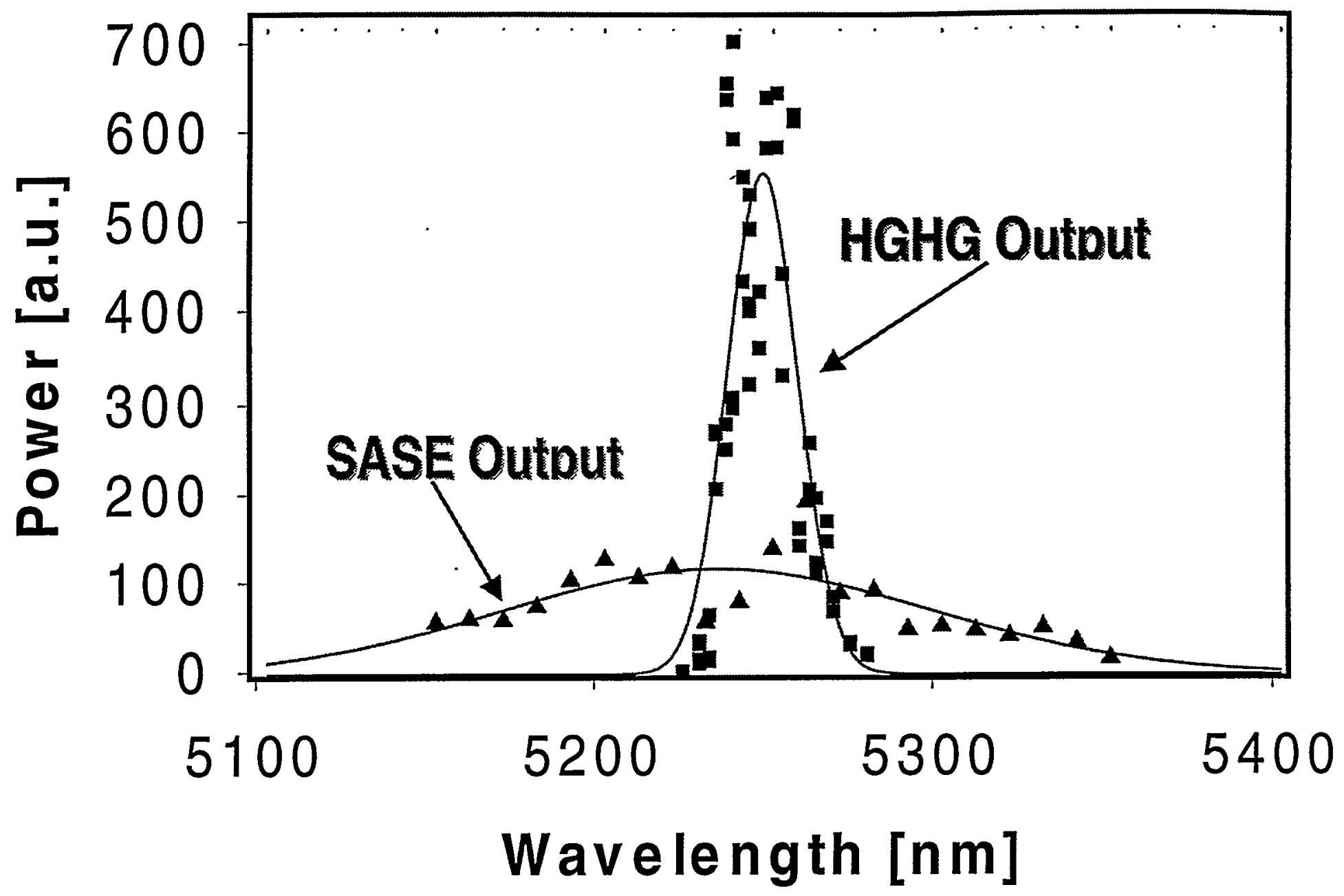

Figure 11 


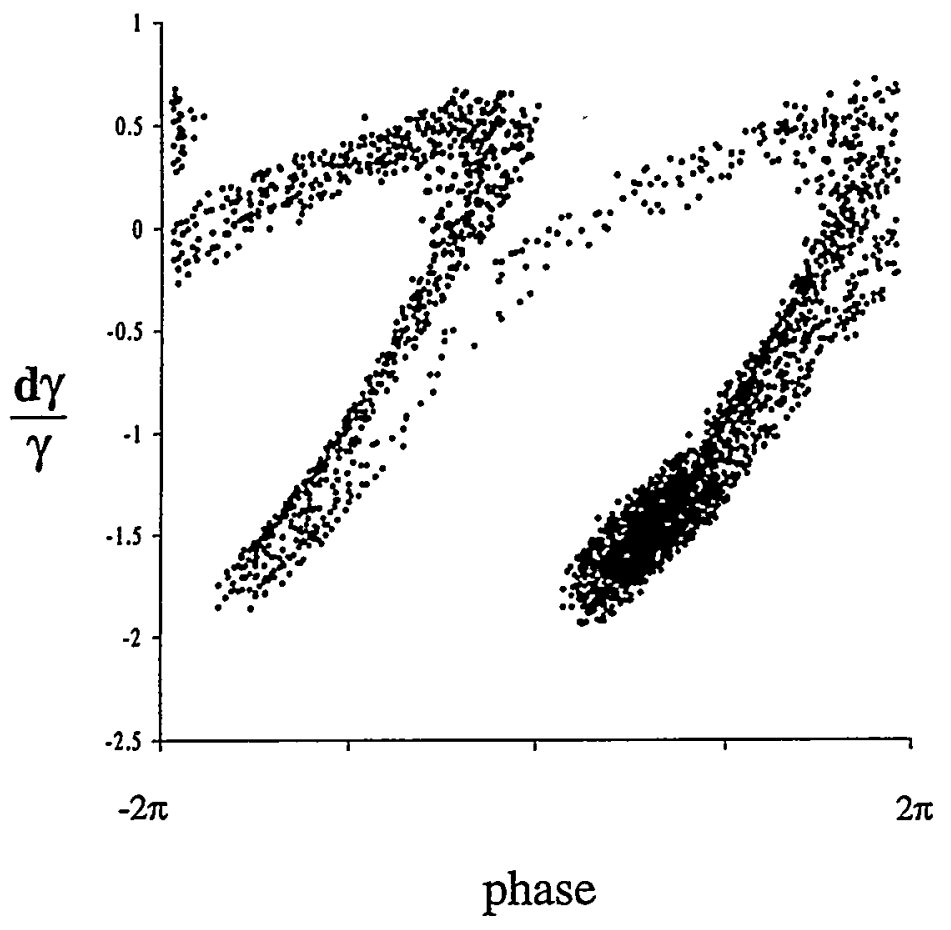

Figure 12 
HGHG pulse energy vs. CO2 power

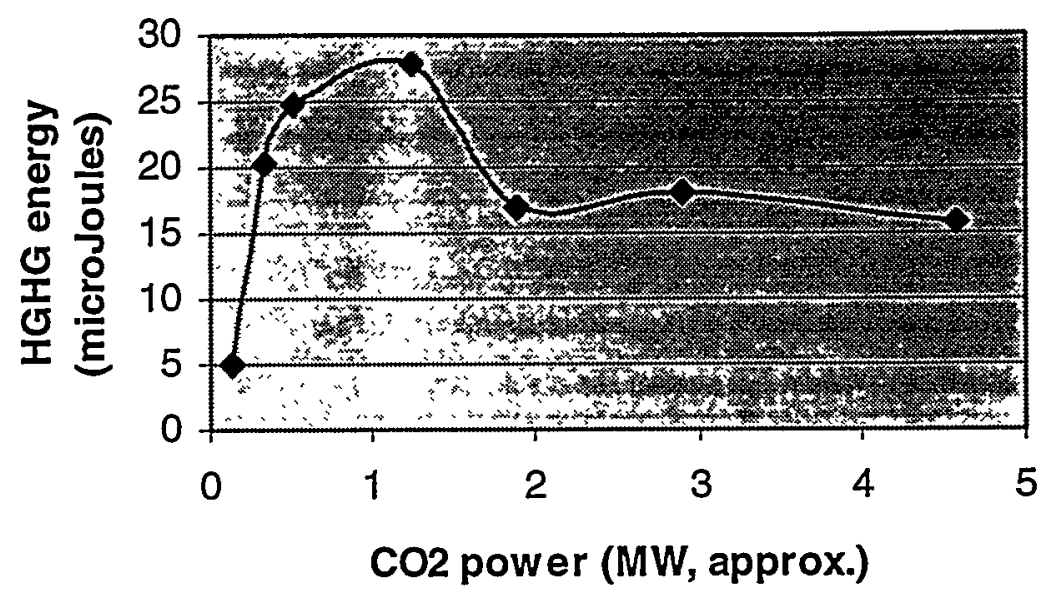

\section{Simulation: HGHG vs. $\mathrm{CO} 2$ power}

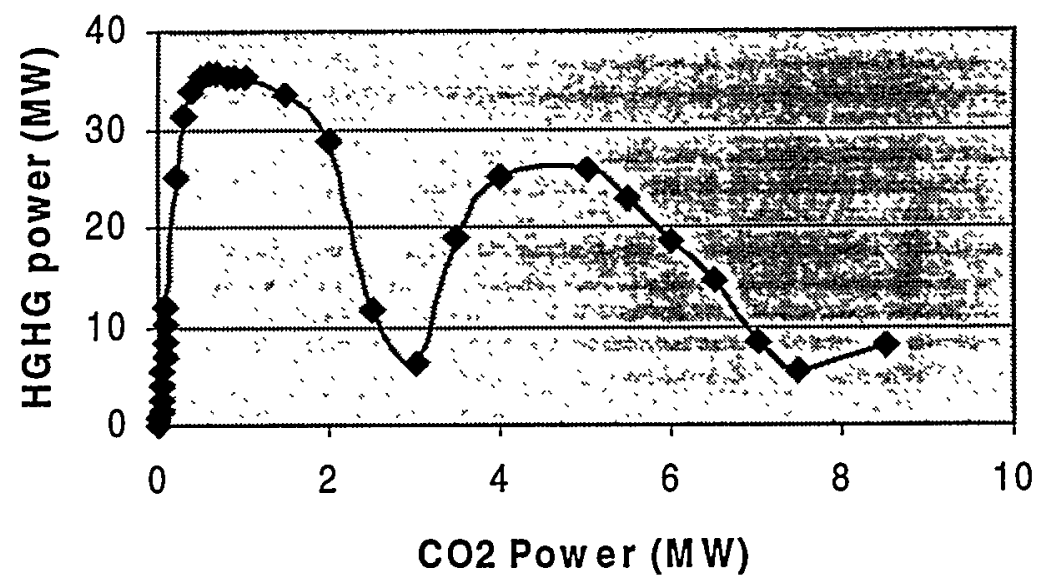

Figure 13 